Audiology

Neurotology
Audiol Neurotol 2014;19:41-48

DOI: $10.1159 / 000356174$
Received: May 28, 2013

Accepted after revision: October 4, 2013

Published online: December 11, 2013

\title{
Separating the Contributions of Olivocochlear and Middle Ear Muscle Reflexes in Modulation of Distortion Product Otoacoustic Emission Levels
}

\author{
Nikolaus E. Wolter ${ }^{a}$ Robert V. Harrison ${ }^{a, b}$ Adrian L. James ${ }^{a, c}$ \\ a Department of Otolaryngology, Head and Neck Surgery, University of Toronto, bepartment of Otolaryngology \\ and Program in Neuroscience and Mental Health, Hospital for Sick Children, and ' Department of Otolaryngology, \\ Hospital for Sick Children, University of Toronto, Toronto, Ont., Canada
}

\section{Key Words}

Medial olivocochlear system · Middle ear muscle reflex .

Otoacoustic emissions

\begin{abstract}
Objectives: Mediated by the medial olivocochlear system (MOCS), distortion product otoacoustic emission (DPOAE) levels are reduced by presentation of contralateral acoustic stimuli. Such acoustic signals can also evoke a middle ear muscle reflex (MEMR) that also attenuates recorded DPOAE levels. Our aim is to clearly differentiate these two inhibitory mechanisms and to analyze each separately, perhaps allowing the development of novel tests of hearing function. Methods: DPOAE were recorded in real time from chinchillas with normal auditory brainstem response thresholds and middle ear function. Amplitude reduction and its onset latency caused by contralateral presentation of intermittent narrow-band noise (NBN) were measured. Stapedius and tensor tympani muscle tendons were divided without disturbing the ossicular chain, and DPOAE testing was repeated. Results: Peak reduction of $\left(2 f_{1}-f_{2}\right)$ DPOAE levels occurred when the center frequency of contralateral NBN approximated the primary tone $f_{2}$, indicating an $\mathrm{f}_{2}$-frequency-specific response. For a $4.5-\mathrm{kHz}$ centered NBN, DPOAE $\left(f_{2}=4.4 \mathrm{kHz}\right)$ inhibition was $0.1 \mathrm{~dB}(p<0.001)$. This response remained present after tendon division, consistent with an MOCS origin. Low-frequency NBN (center frequency: $0.5 \mathrm{kHz}$ ) reduced
\end{abstract}

otoacoustic emission levels $(0.1 \mathrm{~dB}, \mathrm{p}<0.001)$ across a wide range of DPOAE frequencies. This low-frequency response was abolished by division of the middle ear muscle tendons, clearly indicating MEMR involvement. Conclusions: Following middle ear muscle tendon division, DPOAE inhibition by contralateral stimuli approximating the primary tone $\mathrm{f}_{2}$ persists, whereas responses evoked by lower contralateral frequencies are abolished. This distinguishes the different roles of the MOCS ( $f_{2}$ frequency specific) and MEMR (low frequency only) in contralateral modulation of DPOAE. This analysis helps clarify the pathways involved in an objective test that might have clinical benefit in the testing of neonates.

(c) 2013 S. Karger AG, Basel

\section{Introduction}

The benefits of early detection, intervention and habilitation of children with congenital hearing loss are well established [Yoshinaga-Itano, 2003; Kennedy and McCann, 2004; Kennedy et al., 2005; Morton and Nance, 2006]. However, there are limitations with currently available screening methods such as auditory brainstem response (ABR) and otoacoustic emissions [James, 2011]. This was recognized by the Joint Committee on Infant Hearing [American Academy of Pediatrics and Joint Committee on Infant Hearing, 2007], which emphasized the need for a rapid, reliable screening test that could

\begin{tabular}{ll}
\hline KARGER & $\begin{array}{l}\text { C } 2013 \text { S. Karger AG, Basel } \\
\text { 1420-3030/13/0191-0041 } \$ 38.00 / 0 \quad \text { Karger }\end{array}$ \\
$\begin{array}{l}\text { E-Mail karger@karger.com } \\
\text { www.karger.com/aud }\end{array}$ & $\begin{array}{l}\text { This is an Open Access article licensed under the terms of the } \\
\text { Creative Commons Attribution-NonCommercial 3.0 Un- } \\
\text { ported license (CC BY-NC) (www.karger.com/OA-license), } \\
\text { applicable to the online version of the article only. Distribu- } \\
\text { tion permitted for non-commercial purposes only. }\end{array}$
\end{tabular}

Nikolaus Ernst Wolter, MD

Otolaryngology - Head and Neck Surgery, Hospital for Sick Children

555 University Avenue, Elm Wing 6103-D

Toronto, ON M5G 1X8 (Canada)

E-Mail nikolaus.wolter@mail.utoronto.ca 
identify different types of hearing loss, in particular auditory neuropathy spectrum disorder or other retrocochlear lesions. Contralateral suppression/inhibition of otoacoustic emissions has been identified as a potential method for probing both cochlear and neural function [Moulin et al., 1993; James, 2011; Wagner and Heyd, 2011] and the feasibility of testing in neonates has been demonstrated by our group and others [Abdala et al., 1999; Abdala, 2001; James et al., 2002; Chabert et al., 2006; James, 2011; Abdala et al., 2013].

Distortion product otoacoustic emissions (DPOAE) are small, detectable acoustic signals generated because of nonlinear outer hair cell mechanical responses to the simultaneous presentation of two tonal stimuli. These emissions can be detected in almost all normal-hearing neonates and have been widely studied in humans and other mammals [Moulin et al., 1993; Guinan, 2006; James, 2011]. Contralateral suppression/inhibition of DPOAE (CS-DPOAE) by the application of a contralateral acoustic stimulus is facilitated by the medial olivocochlear system (MOCS) [Fex, 1962; Siegel and Kim, 1982; Guinan, 2006]. This neural pathway is schematically shown in figure 1 (dashed line). Activation of this pathway ultimately leads to inhibition of the outer hair cell activity, and inhibition of DPOAE signals. A note on terminology: the modulation of DPOAE by the MOCS is commonly referred to as 'suppression' when it results in a reduction in DPOAE level [Abdala, 2001; James et al., 2002; Jacobson et al., 2003; James et al., 2005; James, 2011]. Considering that the MOCS can both suppress and enhance DPOAE, the effects are sometimes referred to as 'modulations' [Harrison et al., 2008]. However, Guinan [2010] recommended that as these reductions are due to medial olivocochlear synaptic effects rather than two-tone suppression, the reductions are more aptly referred to as inhibitions'. It is definitely appropriate to use the term 'inhibition' when the mechanisms involved are known to be primarily synaptic. However, when DPOAE signal changes are due to some imposed attenuation factor such as middle ear muscle contraction, 'suppression' might be a better descriptor. It is difficult here to assign the most appropriate label, given that this study is an attempt to disambiguate these two effects. Therefore, we have elected to use the general term 'inhibition' for most of the remainder of this manuscript.

Unilateral high-intensity acoustic stimulation can also trigger contraction of the stapedius muscle in both the ipsilateral and contralateral ear via the middle ear muscle reflex (MEMR) [Mukerji et al., 2010] (fig. 1, solid line). This reflex could also contribute to CS-DPOAE because

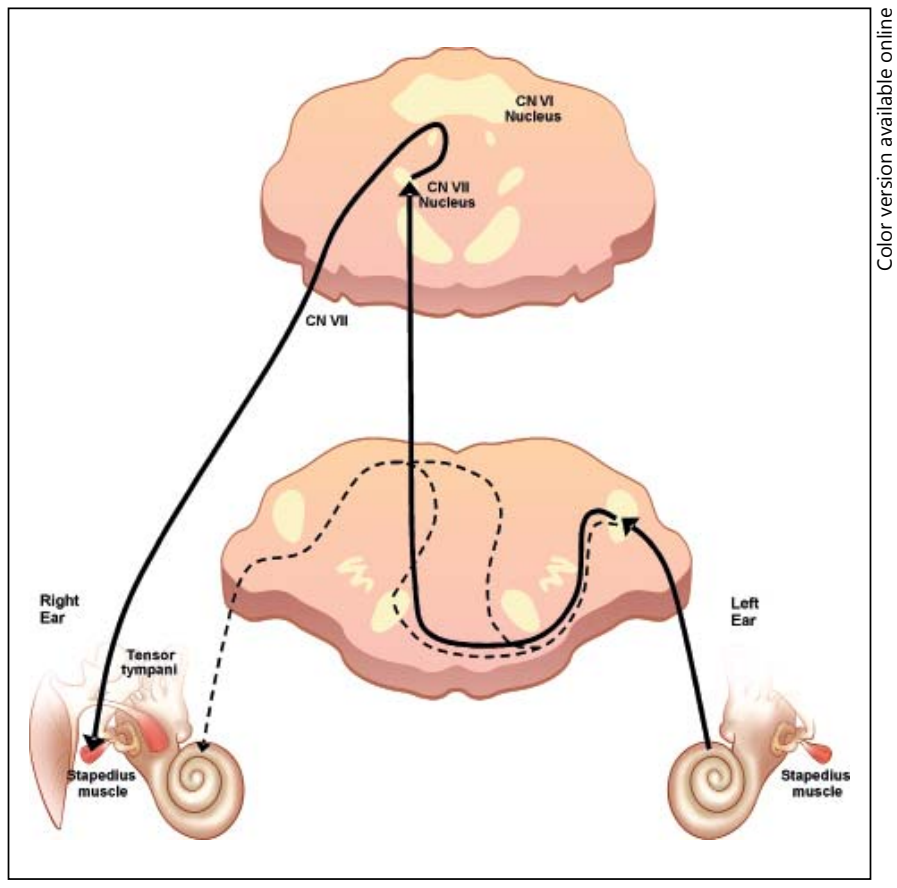

Fig. 1. Middle ear muscle reflex (MEMR) and MOCS pathways (contralateral pathways only). Sound stimulation of the left cochlea activates MEMR pathways (continuous lines) to cause contralateral stapedius muscle contraction. Also shown is the tensor tympani tendon, which may play a role in the MEMR in animals and is innervated by the trigeminal nerve (not shown) [Mukerji et al., 2010]. Sound stimulation can activate the ipsi- and contralateral superior olivary complex and then medial olivocochlear neurons (dashed lines) that modulate cochlear hair cell function [Guinan, 2006]. $\mathrm{CN}=$ Cranial nerve.

traction on the stapes results in stiffening of the ossicular chain and a reduction in middle ear compliance [Pang and Peake, 1986]. When measured with tympanometry, the threshold of this response is around $80 \mathrm{~dB}$ HL using broad-band noise [Moulin et al., 1993]. However, smaller stimuli could conceivably alter middle ear muscle tone sufficiently to cause a small inhibition of the otoacoustic emission level without a detectable change in compliance. Indeed, Neumann et al. [1996] used DPOAE measurement to detect the acoustic reflex and found thresholds for the reflex to be $8 \mathrm{~dB}$ lower than when detected with compliance measurement. A recent report indicates that the acoustic reflex threshold is lower in neonates than adults [Mazlan et al., 2009]. In many animal species (though not in humans), the tensor tympani muscle is also implicated in the acoustic reflex [Ferraro et al., 1981; Relkin et al., 2005; Mukerji et al., 2010].

The inability to differentiate between the suppressive effects of the MOCS and the MEMR pathway has been a 


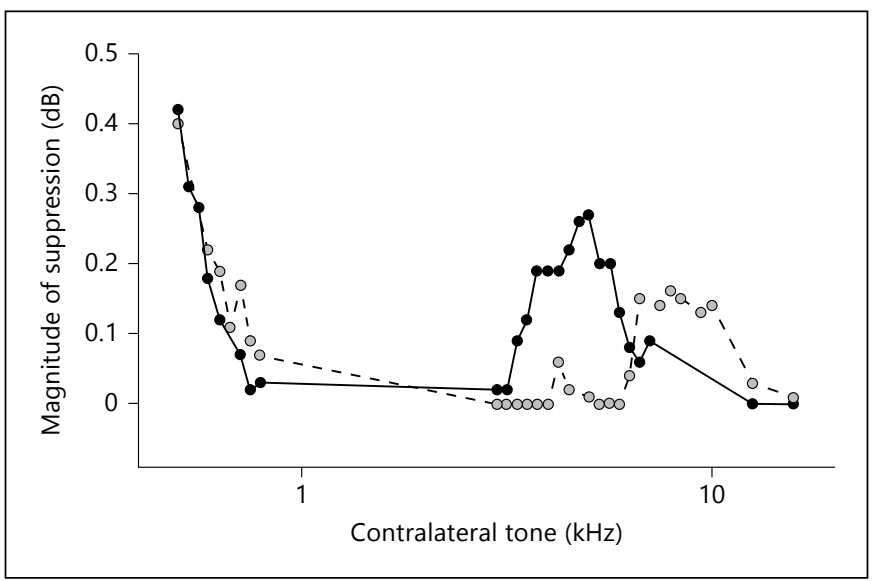

Fig. 2. Contralateral suppression of DPOAE shows frequency specificity, but this is lost for low-frequency stimuli. Real-time DPOAE were recorded at $2 f_{1}-f_{2}$ from primary tones which were set at $f_{2} / f_{1}=$ 1.22 for specific frequencies between $0.6-17 \mathrm{kHz}$ and intensity levels of $\mathrm{L}_{1}=65 \mathrm{~dB}$ SPL and $\mathrm{L}_{2}=55 \mathrm{~dB}$ SPL [Wolter et al., 2012]. In this figure, only 2 frequencies, $\mathrm{f}_{2}=4.4 \mathrm{kHz}$ (black line) and $\mathrm{f}_{2}=7.7$ $\mathrm{kHz}$ (dashed line), are shown for the purposes of clarity. All frequencies can be seen in Wolter et al. [2012]. The magnitude of suppression was greatest when the contralateral tone approximated the $\mathrm{f}_{2}$ frequency. At low contralateral stimulus frequencies, DPOAE suppression was observed regardless of the $f_{2}$ frequency.

disincentive to adopt CS-DPOAE testing in neonates [Sun, 2008]. CS-DPOAE has been shown in both humans and animals to demonstrate some degree of tonotopic behavior. In other words, neural pathways between the ears roughly link equivalent cochleotopic regions. It is assumed that MEMR-related inhibition would not exhibit such properties [Warren and Liberman, 1989]. Work done in our laboratory using real-time measurement of CS-DPOAE in chinchillas has demonstrated that the greatest magnitude of inhibition is achieved when the contralateral tone approximates the DPOAE $\mathrm{f}_{2}$ stimulus frequency when specific frequencies were used in the range from 1.6 to $7.7 \mathrm{kHz}$, confirming that CS-DPOAE shows $\mathrm{f}_{2}$ frequency specificity [Wolter et al., 2012]. Previously unpublished data in figure 2 demonstrate that contralateral inhibition of DPOAE at $\mathrm{f}_{2}=4.4 \mathrm{kHz}$ is greatest when the contralateral tone approximates $\mathrm{f}_{2}=4.4 \mathrm{kHz}$ (black symbols). The gray symbols show equivalent findings for DPOAE and contralateral tones centered at $\mathrm{f}_{2}=7.7 \mathrm{kHz}$. However, when low-frequency tones were applied to the contralateral ear, we also measured a significant amount of DPOAE inhibition. Given that low-frequency, high-intensity tones can stimulate the MEMR and attenuate DPOAE signals, we hypothesized that the low-frequency inhibition is due to the effects of the MEMR, whereas the $\mathrm{f}_{2}$-frequency-specific inhibition is due to the MOCS. In the present study we tested this hypothesis with an animal model in which CS-DPOAE was assessed before and after dividing middle ear muscle tendons in the test ear.

\section{Materials and Methods}

Adult chinchillas (Chinchilla lanigera) were the animal models of choice because of their large bulla cavities and consequent ease of surgical access to middle ear structures. For all procedures, the animals were anesthetized with intraperitoneal ketamine $(15 \mathrm{mg} /$ $\mathrm{kg}$ ) and xylazine $(2.5 \mathrm{mg} / \mathrm{kg})$. Additional one-half doses of anesthetic were administered hourly and as required based on muscle tone and respiratory pattern. Each animal was anesthetized and tested on 2 separate occasions, the first to check ABR, MEMR and CS-DPOAE, the second after a 2 -week interval to perform surgical division of middle ear muscle tendons and to repeat CS-DPOAE and MEMR testing. A 2-week interval was found to reduce stress in the animals and therefore anesthetic requirements in the second surgery. All testing was performed within a sound-attenuating chamber. Approval for this study was granted based on the guidelines of the Canadian Council on Animal Care.

\section{Baseline Hearing and MEMR Testing}

The initial auditory evaluation comprised ABR, tympanometry and real-time DPOAE measurement. For ABR testing, electrodes were placed in a mastoid/bulla-to-vertex configuration. Stimuli were presented in a closed system using Etymotic ER-2 transducers (Etymotic Research, Elk Grove Village, Ill., USA). ABR signals were amplified $(\times 1,000)$, and filtered $(100-1,500 \mathrm{~Hz})$ and averaged (SmartEP system; Intelligent Hearing Systems, Miami, Fla., USA). Normal auditory thresholds were confirmed by measuring ABR to tonal stimuli from 0.5 to $16 \mathrm{kHz}$, and only animals with thresholds below $20 \mathrm{~dB}$ SPL were included. Compliance testing (Zodiac 901; Madsen Electronics, Taastrup, Denmark) was used to confirm the presence of a measurable MEMR in each chinchilla at stimulus intensities of 60-90 dB SPL.

\section{Real-Time DPOAE Measurement}

Real-time DPOAE were recorded from the test ear (chosen randomly), using a customized research device (Vivo 600 DPR; Vivosonic, Toronto, Ont., Canada) while an intermittent narrow-band noise (NBN) stimulus was applied to the contralateral ear. The distortion product recorded was the $2 f_{1}-f_{2}$, from primary tones at an $\mathrm{f}_{2}$ of $4.4 \mathrm{kHz}\left(\mathrm{f}_{2} / \mathrm{f}_{1}=1.22\right)$ and intensity levels of $\mathrm{L}_{1}=65 \mathrm{~dB}$ SPL and $\mathrm{L}_{2}=55 \mathrm{~dB}$ SPL. We concentrated on these particular stimuli as they had been found to evoke the maximum response in our previous work in chinchillas [Wolter et al., 2012]. The levels chosen to give optimal DPOAE were determined from input/output functions of primary level versus signal level. For this experiment we focused on $\mathrm{f}_{2}=4.4 \mathrm{kHz}$ because it was the most robust and reliable response in our previous work with the chinchilla [Wolter et al., 2012].

\section{Contralateral Acoustic Stimulus}

An intermittent NBN was applied to the contralateral ear using an Etymotic ER-2 transducer (Etymotic Research) with a foam ear insert. A 550-ms white-noise stimulus was repeated every $1,223 \mathrm{~ms}$ (Cool Edit 2000; Syntrillium Software Corporation, Phoenix, 

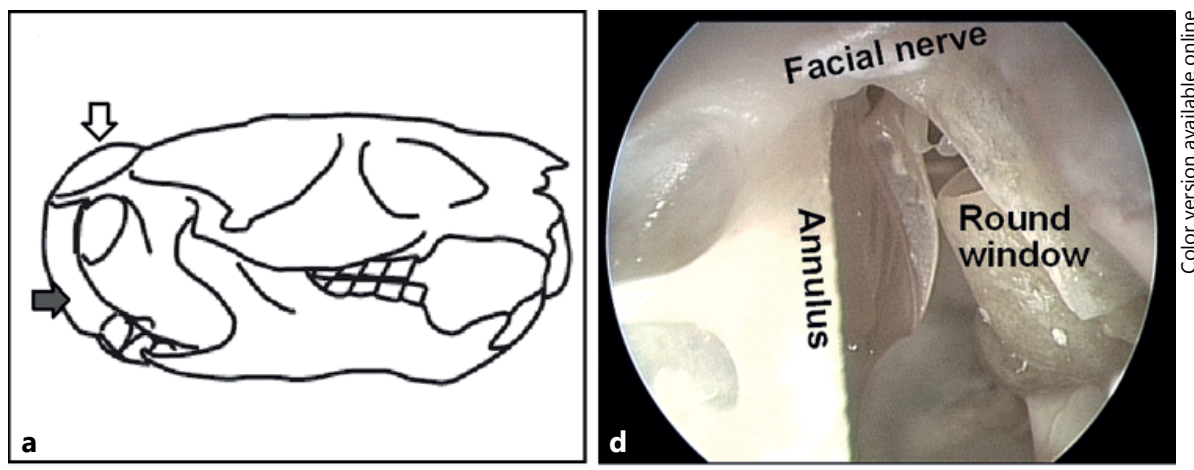

Fig. 3. Surgical approaches to middle ear muscle division. a Schematic diagram of the skull of an adult Chinchilla lanigera. The tensor tympani tendon was accessed using a superior approach through the superior tympanic bulla (white arrow). The stapedius tendon was accessed through the inferior tympanic bulla (gray arrow). Running in between these is the transverse sinus (asterisk). b, c The tensor tympani tendon is seen (thick black arrow) intact (b) and then divided (c). $\mathbf{d}$ Initial view once the middle ear has been accessed via the posterior approach. The incudostapedial joint is visible; however, the stapedius is obscured by a small boney wall. e Curettage of the boney wall allows access to the stapedius tendon (thin arrow). f Stapedius tendon reflected off the bone. Note the incudostapedial joint remains intact.
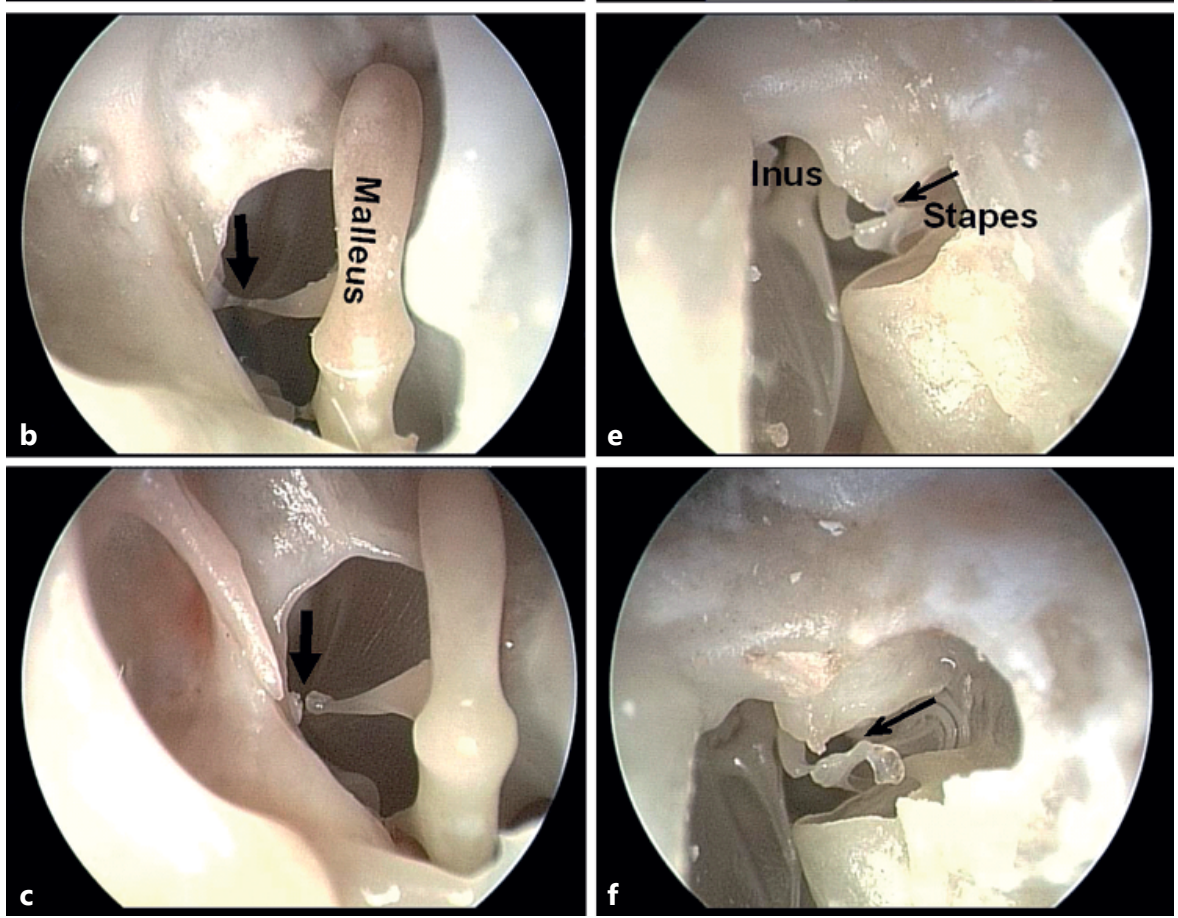

Ariz., USA). The noise was passed through a dual-channel filter (Stanford Research Systems, Sunnyvale, Calif., USA) to create the narrow-band signals. These were presented at intensities of $75 \mathrm{~dB}$ SPL so as to ensure adequate stimulation of the MEMR. In this experiment we used stimuli with center frequencies of $500 \mathrm{~Hz}$ and $4.5 \mathrm{kHz}$, with a bandwidth of $500 \mathrm{~Hz}$. These parameters were chosen as optimal on the basis of previous findings [Wolter et al., 2012]. Measurements were made before and after the muscle division described below. Calibration was performed in a 1-ml sound tube using a sound level meter (Larson Davis Model 831; Larson Davis, Depew, N.Y., USA) and a standardized tone generator (CRL $511 \mathrm{D}$ calibrator; Cirrus Research Ltd, Hunmanby, UK).

\section{Middle Ear Muscle Tendon Division}

On the day of surgery, DPOAE were remeasured. A curvilinear incision was then made behind the test ear and carried to the level of the mandibular angle to expose the tympanic bullae (fig. 3a). The superior bulla proper (white arrow) was opened using a small rongeur, avoiding the venous sinuses, to expose the tensor tympani tendon (fig. 3b). This was then divided using a myringotomy blade (fig. 3c). The occipital muscle was identified and dissected off the inferior bulla, using a combination of blunt dissection and bipolar diathermy. Once the labyrinthine part of the inferior bulla was exposed (fig. 3a, gray arrow), a rongeur was used to enter the bulla. A septation preventing access to the stapedius tendon (fig. $3 \mathrm{~d}$ ) was carefully curetted with a pick developed from the crimped tip of a 21-gauge needle, taking care not to damage the annulus or ossicles beyond the septation (fig. 3e). Once the stapedius muscle was identified, a tool developed from a 25-gauge needle was used to reflect the stapedius muscle from its bony attachment without damaging the ossicular chain (fig. 3f). The stapes footplate was kept in view during this maneuver, and if any movement was seen, the procedure was terminated. Meticulous hemostasis was maintained with bipolar diathermy to prevent bleeding into the middle ear. The wound was closed in layers to seal the middle ear space. The ear canal and tympanic membrane were not disturbed by this approach to the middle ear. Following this surgery, tympanometry was used to confirm abolition of the MEMR in the test ear. The DPOAE probe was then replaced in the test ear canal, and DPOAE levels were then recorded with contralateral stimulation as described above. 


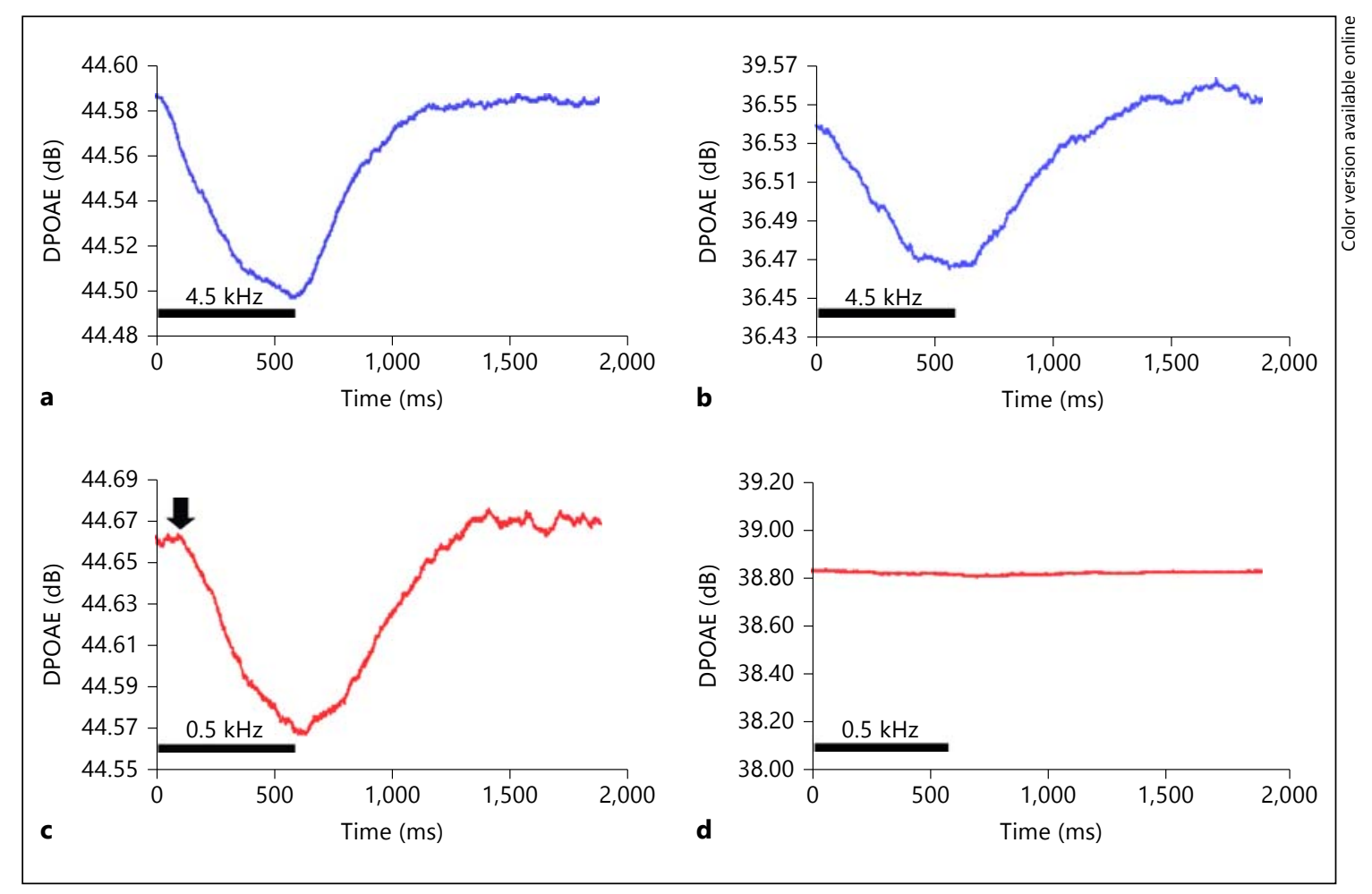

Fig. 4. Averaged DPOAE signals recorded over a 2-second period with a 550-ms contralateral sound stimulus (black bar). DPOAE were recorded at $\mathrm{f}_{2}=4.4 \mathrm{kHz}$ and intensity levels of $\mathrm{L}_{1}=65 \mathrm{~dB}$ SPL and $\mathrm{L}_{2}=55 \mathrm{~dB}$ SPL. Suppression of DPOAE was induced with the contralateral signal at either $4.5 \mathrm{kHz}(\mathbf{a}, \mathbf{b})$ or $0.5 \mathrm{kHz}(\mathbf{c}, \mathbf{d})$ at intensity levels of $75 \mathrm{~dB}$ SPL. a Contralateral noise signal cen-

\section{Analysis of Results}

Real-time DPOAE signal traces were examined for evidence of level inhibition synchronized with the application of the contralateral acoustic stimulus [James et al., 2005]. For an improvement in signal-to-noise ratio, postanalytic signal averaging was performed. A contralateral inhibitory response was defined as a statistically significant reduction in DPOAE level during contralateral stimulus presentation. The inhibition onset latency was the time interval between the onset of the acoustic stimulus and the onset of the DPOAE inhibition. This was accurately quantified from the intersection of lines plotted through the baseline DPOAE level and the first part of the inhibition response. Offset latency was measured in a similar fashion. Measurement of DPOAE inhibition characteristics was made while blinded to the contralateral stimulus parameters.

Statistical tests were performed using SPSS software (SPSS version 16.0; SPSS Inc., Chicago, Ill., USA). Significance was determined by $\mathrm{p}<0.05$. Paired data were compared using Student's $\mathrm{t}$ test ( $\alpha$ set at 0.05 ). Approximately 4 weeks after the studies' completion, the recordings were reanalyzed to determine intraobserver reliability. All identifying information was removed from the recordings so as to mask the investigator regarding the animal as well as pre- and postoperative status. An interclass correlation coefficient was calculated to determine intraobserver reliability of measurements.

Middle Ear Muscle Contribution to DPOAE Suppression Reflex tered at $4.5 \mathrm{kHz}$; DPOAE changes prior to middle ear muscle tendon division. $\mathbf{b} 4.5-\mathrm{kHz}$ stimulus; DPOAE suppression after middle ear muscle tendon division. c Contralateral noise centered on $0.5 \mathrm{kHz}$; DPOAE prior to middle ear muscle tendon division. d $0.5-\mathrm{kHz}$ stimulus; DPOAE after middle ear muscle tendon division.

\section{Results}

Six animals fit our ABR threshold criterion for inclusion and had clearly demonstrable preoperative contralateral inhibition of DPOAE and MEMR. One subject was lost to anesthesia-related problems, 1 animal developed an infection preoperatively and 1 chinchilla had a failed surgery when the incudostapedial joint became dislocated in the process of removing the bony septation. Three chinchillas went on to have successful middle ear muscle tendon divisions and complete DPOAE recording.

The tracings shown in figure 4 are typical examples of the averaged DPOAE level during presentation of an intermittent contralateral stimulus (see also online suppl. fig. 1; for all online suppl. material, see www.karger.com/ doi/10.1159/000356174). All are obtained from 3-min recordings with $\mathrm{f}_{2}=4.4 \mathrm{kHz}$ and a contralateral NBN stimulus of $60 \mathrm{~dB}$. The traces were obtained before (fig. $4 \mathrm{a}, \mathrm{c}$ ) and after (fig. 4b, d) middle ear muscle tendon division. Responses to contralateral stimulation with $4.5-\mathrm{kHz}$ NBN 
Table 1. Inhibition curve characteristics

\begin{tabular}{|c|c|c|c|c|c|c|}
\hline & $\mathrm{C}-\mathrm{NBN}$ at $0.5 \mathrm{kHz}$ & $\mathrm{C}-\mathrm{NBN}$ at $4.5 \mathrm{kHz}$ & $\mathrm{p}$ & $\mathrm{C}-\mathrm{NBN}$ at $0.5 \mathrm{kHz}$ & $\mathrm{C}-\mathrm{NBN}$ at $4.5 \mathrm{kHz}$ & $\mathrm{p}$ \\
\hline Mean onset latency $\pm \mathrm{SD}, \mathrm{ms}$ & $96 \pm 16$ & $37 \pm 15$ & 0.03 & $0 \pm 0$ & $60 \pm 5$ & 0.002 \\
\hline Mean offset latency $\pm \mathrm{SD}, \mathrm{ms}$ & $645 \pm 31$ & $605 \pm 16$ & 0.25 & $0 \pm 0$ & $615 \pm 57$ & 0.003 \\
\hline
\end{tabular}

$\mathrm{C}-\mathrm{NBN}=$ Contralateral NBN.

(centered near to DPOAE primary frequency $f_{2}$ ) are shown in the upper panels (fig. $4 \mathrm{a}, \mathrm{b}$ ), and responses to the contralateral NBN centered at $0.5 \mathrm{kHz}$ are shown below (fig. 4c, d). Before tendon division, mean DPOAE levels were suppressed by $0.1 \mathrm{~dB}$ by a contralateral 4.4$\mathrm{kHz}$ NBN (fig. 4a), a statistically significant level of DPOAE inhibition from the baseline (table 1). Following tendon division, the mean DPOAE inhibition decreased to $0.08 \mathrm{~dB}$ (fig. $4 \mathrm{~b})$ but remained significant $(\mathrm{p}<0.05)$. Using low-frequency contralateral NBN (centered at 0.5 $\mathrm{kHz}$ ), the mean magnitude of inhibition before tendon division was $0.104 \mathrm{~dB}$ (fig. 4c). Following division of the middle ear muscle tendons, contralateral inhibition of the DPOAE was no longer detectable with low-frequency contralateral NBN (fig. 4d).

Prior to tendon division, the onset latency of inhibition was significantly longer (table 1 ) for low-frequency inhibition evoked by the low-frequency NBN (96 ms) than the $\mathrm{f}_{2}$-frequency-specific response to $4.5-\mathrm{kHz} \mathrm{NBN}$ (37 ms; $\mathrm{p}<0.05)$. This difference in onset latency can be seen in the DPOAE example of figure 4 (c, arrow). After dividing the tendons there was an increase in the onset latency of CS-DPOAE from NBN at $4.5 \mathrm{kHz}$ from 37 to $60 \mathrm{~ms}$ (table 1); however, this change was not found to be statistically significant $(\mathrm{p}=0.153)$.

Inhibition of DPOAE persists for a period of time following cessation of the contralateral stimulation before returning to baseline, and this was defined as the offset latency. Prior to tendon division, the offset latency was longer in response to low-frequency contralateral NBN than to contralateral NBN at $\mathrm{f}_{2}$ (645 vs. $605 \mathrm{~ms}$ ), but this was not statistically significant $(\mathrm{p}=0.25)$. After tendon division, the offset latency from contralateral NBN at $\mathrm{f}_{2}$ increased to $615 \mathrm{~ms}$, but this was not significantly longer than the preoperative value $(\mathrm{p}=0.75)$.

Intraobserver reliability was assessed by repeating the measurements at a later date while being masked to the previous measurements and conditions in all chinchillas.
The intraobserver reliability was very high, with a correlation coefficient of $0.99(\mathrm{p}<0.0001)$ for the magnitude of inhibition, $0.87(\mathrm{p}<0.0001)$ for the onset latency, and 0.98 for the offset latency $(\mathrm{p}<0.0001)$.

\section{Discussion}

Since the initial observations of CS-DPOAE there has been some controversy over the extent of the contribution from MOCS and MEMR [Moulin et al., 1993; Goodman and Keefe, 2006]. Previous animal studies of the olivocochlear reflex have sought to exclude a middle ear muscle effect by dividing the stapedius and tensor tympani muscles. For example, Puel and Rebillard [1990] reported that it was possible to measure inhibition of DPOAE by contralateral broad-band noise following division of the stapedius and tensor tympani in guinea pigs. Liberman [1989] found that contralateral inhibition of the compound action potential in 5 cats was identical to that in a cat with divided middle ear muscles. Maison et al. [2012] pharmacologically paralyzed the stapedius muscle in mice and were able to observe contralateral inhibition using broad-band noise. In contrast, sectioning of olivocochlear neurons by division of the ipsilateral inferior vestibular nerve abolished CS-DPOAE (MEMR are not affected by this intervention as the stapedius muscle is innervated by a branch of the facial nerve, and the tensor tympani by a branch of the trigeminal nerve). Furthermore, the olivocochlear reflex has been demonstrated in humans with absent acoustic reflexes (with facial nerve palsy or following surgical section of the stapedius) [Moulin et al., 1993] and is absent following vestibular neurectomy, in which the olivocochlear bundle is divided [Giraud et al., 1997].

A full understanding of the mechanisms involved in contralateral inhibition of DPOAE is confounded by the simultaneous activation of these two pathways. In this re- 
gard, Büki and others found significant differences in DPOAE level and phase using high- and low-level contralateral acoustic stimulation [Giraud et al., 1997; Büki et al., 2000]. These findings were consistent with modeling of stapedial annular ligament tension and showing that the acoustic reflex causes maximal DPOAE inhibition and phase change at low DPOAE frequencies (i.e. $<1 \mathrm{kHz}$ ).

In the present study we have shown that division of the middle ear muscle tendons in the chinchilla clearly allows the differentiation of the contribution of the MEMR from that of the MOCS in CS-DPOAE. Using a well-established method of real-time DPOAE to detect contralateral inhibition [Kemp, 1979; Liberman, 1989; Puel and Rebillard, 1990; James, 2011; Wolter et al., 2012], we have shown that low-frequency $(<1 \mathrm{kHz}) \mathrm{NBN}$ suppresses DPOAE regardless of the primary frequency. This response to low-frequency stimulation is abolished by division of the middle ear muscle tendons and therefore is mediated wholly or in part by the MEMR. In contrast, higher-frequency $(>1 \mathrm{kHz})$ NBN causes maximal contralateral inhibition when the stimulus is centered at a frequency close to DPOAE $\mathrm{f}_{2}$. This $\mathrm{f}_{2}$-frequency-specific response is not significantly altered by dividing the tendons of the middle ear muscles and is characteristic of MOCS activity. Importantly, we show that the DPOAE inhibition onset latency is longer in the case of MEMR involvement (96 ms) compared with the MOCS-mediated reflex (37 ms). This is consistent with the longer neural pathways and the myogenic components involved in the MEMR-mediated DPOAE attenuation. The measured effect of the MOCS on DPOAE is a combined result of the interference of these two distortion and reflection components which can interact constructively or destructively within the ear canal [Shera and Guinan, 1999; Guinan, 2010]. Studies that have separated the distortion and reflection components using fine-frequency steps in humans have demonstrated that medial olivocochlear stim- ulation inhibits both components and shifts their phase with the reflection component being more affected than the distortion component [Abdala et al., 2009; Deeter et al., 2009]. These differential effects may make the interpretation of DPOAE inhibition more difficult if the sources are not separated. In the present study, identical conditions were tested before and after middle ear muscle tendon division, thus controlling for the differential effects of the distortion and reflection components.

Since CS-DPOAE can be detected in normally hearing infants, it has potential as a clinically useful test. It could provide an objective, $\mathrm{f}_{2}$-frequency-specific assessment of hearing threshold [Wolter et al., 2012]. As indicated by the 2007 Joint Committee on Infant Hearing, this would be of great benefit in newborn hearing screening [American Academy of Pediatrics and Joint Committee on Infant Hearing, 2007] and may facilitate early diagnosis and prognostication in neonatal auditory neuropathy spectrum disorder [James, 2011]. It may be of some value to discriminate between and separately assess the MEMR and MOCS contributions, with low-frequency contralateral stimuli for the former and stimuli close to $f_{2}$ for the latter. Our real-time DPOAE recording technique also provides the opportunity to separately compare the dynamic aspects of MEMR and MOCS components (e.g. onset latency and time constants) that might have some diagnostic value.

\section{Acknowledgments}

Financial and material support: Canadian Institutes of Health Research; the Hearing Foundation of Canada; and the University of Toronto Connaught Fund.

\section{Disclosure Statement}

None.

\section{References}

Abdala C: DPOAE suppression tuning: cochlear immaturity in premature neonates or auditory aging in normal-hearing adults? J Acoust Soc Am 2001;110:3155-3162.

Abdala C, Ma E, Sininger YS: Maturation of medial efferent system function in humans. J Acoust Soc Am 1999;105:2392-2402.

-Abdala C, Mishra S, Garinis A: Maturation of the human medial efferent reflex revisited. J Acoust Soc Am 2013;133:938-950.
Abdala C, Mishra SK, Williams TL: Considering distortion product otoacoustic emission fine structure in measurements of the medial olivocochlear reflex. J Acoust Soc Am 2009;125: 1584-1594.

American Academy of Pediatrics, Joint Committee on Infant Hearing: Year 2007 position statement: principles and guidelines for early hearing detection and intervention programs. Pediatrics 2007;120:898-921.
Büki B, Wit HP, Avan P: Olivocochlear efferent vs middle-ear contributions to the alteration of otoacoustic emissions by contralateral. Brain Res 2000;852:140-150.

Chabert R, Guitton MJ, Amram D, Uziel A, Pujol R, Lallemant JG, Puel JL: Early maturation of evoked otoacoustic emissions and medial olivocochlear reflex in preterm neonates. Pediatr Res 2006;59:305-308.
Middle Ear Muscle Contribution to

DPOAE Suppression Reflex
Audiol Neurotol 2014;19:41-48 DOI: $10.1159 / 000356174$ 
Deeter R, Abel R, Calandruccio L, Dhar S: Contralateral acoustic stimulation alters the magnitude and phase of distortion product otoacoustic emissions. J Acoust Soc Am 2009; 126:2413-2424.

Ferraro JA, Melnick W, Gerhardt KR: Effects of prolonged noise exposure in chinchillas with severed middle ear muscles. Am J Otolaryngol 1981;2:13-18.

Fex J: Auditory activity in centrifugal and centripetal cochlear fibres in cat: a study of a feedback system. Acta Physiol Scand Suppl 1962;189: $1-68$.

- Giraud AL, Wable J, Chays A, Collet L, ChéryCroze S: Influence of contralateral noise on distortion product latency in humans: is the medial olivocochlear efferent system involved? J Acoust Soc Am 1997;102:2219-2227.

Goodman SS, Keefe DH: Simultaneous measurement of noise-activated middle-ear muscle reflex and stimulus frequency otoacoustic emissions. J Assoc Res Otolaryngol 2006;7:125-139.

- Guinan JJ Jr: Olivocochlear efferents: anatomy, physiology, function, and the measurement of efferent effects in humans. Ear Hear 2006;27: 589-607.

- Guinan JJ Jr: Cochlear efferent innervation and function. Curr Opin Otolaryngol Head Neck Surg 2010;18:447-453.

Harrison RV, Sharma A, Brown T, Jiwani S, James AL: Amplitude modulation of DPOAEs by acoustic stimulation of the contralateral ear. Acta Otolaryngol 2008;128:404-407.

Jacobson M, Kim S, Romney J, Zhu X, Frisina RD: Contralateral suppression of distortion-product otoacoustic emissions declines with age: a comparison of findings in CBA mice with human listeners. Laryngoscope 2003;113:17071713.

-James AL: The assessment of olivocochlear function in neonates with real-time distortion product otoacoustic emissions. Laryngoscope 2011;121:202-213.

-James AL, Harrison RV, Pienkowski M, Dajani HR, Mount RJ: Dynamics of real time DPOAE contralateral suppression in chinchillas and humans. Int J Audiol 2005;44:118-129.
James AL, Mount RJ, Harrison RV: Contralateral suppression of DPOAE measured in real time. Clin Otolaryngol Allied Sci 2002;27:106-112.

Kemp DT: Evidence of mechanical nonlinearity and frequency selective wave amplification in the cochlea. Arch Otorhinolaryngol 1979;224: 37-45.

Kennedy C, McCann D: Universal neonatal hearing screening moving from evidence to practice. Arch Dis Child Fetal Neonatal Ed 2004; 89:F378-F383.

Kennedy C, McCann D, Campbell MJ, Kimm L, Thornton R: Universal newborn screening for permanent childhood hearing impairment: an 8-year follow-up of a controlled trial. Lancet 2005;366:660-662.

Liberman MC: Rapid assessment of soundevoked olivocochlear feedback: suppression of compound action potentials by contralateral sound. Hear Res 1989;38:47-56.

Maison SF, Usubuchi H, Vetter DE, Elgoyhen AB, Thomas SA, Liberman MC: Contralateralnoise effects on cochlear responses in anesthetized mice are dominated by feedback from an unknown pathway. J Neurophysiol 2012;108:491-500.

-Mazlan R, Kei J, Hickson L: Test-retest reliability of the acoustic stapedial reflex test in healthy neonates. Ear Hear 2009;30:295-301.

Morton CC, Nance WE: Newborn hearing screening: a silent revolution. $\mathrm{N}$ Engl J Med 2006;354:2151-2164.

-Moulin A, Collet L, Duclaux R: Contralateral auditory stimulation alters acoustic distortion products in humans. Hear Res 1993;65:193-210.

Mukerji S, Windsor AM, Lee DJ: Auditory brainstem circuits that mediate the middle ear muscle reflex. Trends Amplif 2010;14:170-191.

-Neumann J, Uppenkamp S, Kollmeier B: Detection of the acoustic reflex below $80 \mathrm{~dB} \mathrm{HL}$. Audiol Neurotol 1996;1:359-369.

Pang X, Peake W: How do contractions of the stapedius muscle alter the acoustic properties of the ear? In Allen JB, et al (eds): Peripheral Auditory Mechanisms. Proceedings of a conference held at Boston University, Boston, MA, August 13-16, 1985. Berlin, Springer, 1986, pp 36-43.
Puel JL, Rebillard G: Effect of contralateral sound stimulation on the distortion product $2 \mathrm{f}_{1}-\mathrm{f}_{2}$ : evidence that the medial efferent system is involved. J Acoust Soc Am 1990;87:16301635.

Relkin EM, Sterns A, Azeredo W, Prieve BA, Woods CI: Physiological mechanisms of onset adaptation and contralateral suppression of DPOAEs in the rat. J Assoc Res Otolaryngol 2005;6:119-135.

-Shera CA, Guinan JJ Jr: Evoked otoacoustic emissions arise by two fundamentally different mechanisms: a taxonomy for mammalian OAEs. J Acoust Soc Am 1999;105(pt 1):782798.

- Siegel JH, Kim DO: Efferent neural control of cochlear mechanics? Olivocochlear bundle stimulation affects cochlear biomechanical nonlinearity. Hear Res 1982;6:171-182.

Sun XM: Contralateral suppression of distortion product otoacoustic emissions and the middle-ear muscle reflex in human ears. Hear Res 2008;237:66-75.

Wagner W, Heyd A: Measurement of medial olivocochlear efferent activity in humans: comparison of different distortion product otoacoustic emission-based paradigms. Otol Neurotol 2011;32:1379-1388.

-Warren EH 3rd, Liberman MC: Effects of contralateral sound on auditory-nerve responses. II. Dependence on stimulus variables. Hear Res 1989;37:105-121.

-Wolter NE, Harrison RV, James AL: Contralateral suppression of otoacoustic emissions: working towards a simple objective frequency specific test for hearing screening; in $\mathrm{Naz}$ S (ed): Hearing Loss. Rijeka, InTech, 2012, chapt 2, pp 25-50. DOI: 10.5772/34496. http://www.intechopen.com/books/ hearing-loss/contralateral-suppression-ofotoacoustic-emissions-working-towards-asimple-objective-frequency-spec.

Yoshinaga-Itano C: Early intervention after universal neonatal hearing screening: impact on outcomes. Ment Retard Dev Disabil Res Rev 2003;9:252-266. 